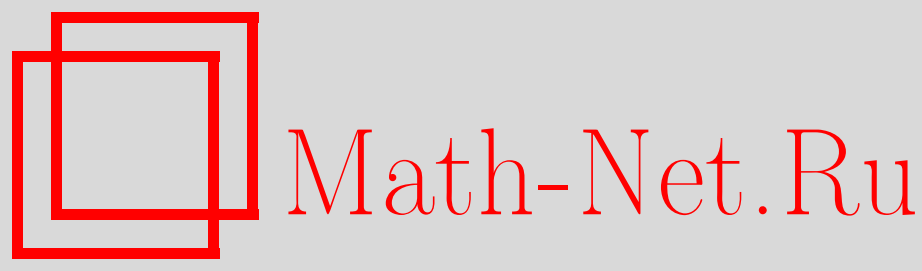

А. В. Юров, В. А. Юров, С. В. Червон, М. Сами, Потенциал полной энергии как суперпотенциал в интегрируемых космологических моделях, ТМФ, 2011, том 166, номер 2, 299-311

DOI: https://doi.org/10.4213/tmf6611

Использование Общероссийского математического портала Math-Net.Ru подразумевает, что вы прочитали и согласны с пользовательским соглашением http: //www . mathnet.ru/rus/agreement

Параметры загрузки:

IP : 54.224 .187 .69

26 апреля 2023 г., 13:43:32

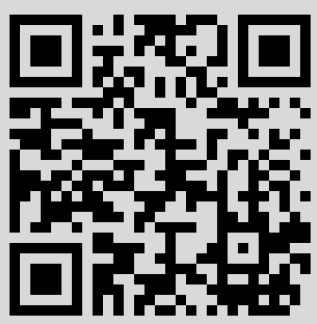




\title{
ПОТЕНЦИАЛ ПОЛНОЙ ЭНЕРГИИ КАК СУПЕРПОТЕНЦИАЛ В ИНТЕГРИРУЕМЫХ КОСМОЛОГИЧЕСКИХ МОДЕЛЯХ
}

\begin{abstract}
Изучен класс интегрируемых потенциалов самодействия, которые удовлетворяют следующим условиям: 1) спонтанно нарушают симметрию; 2) описывают фазу инфляции с естественным выходом (т. е. с выходом из инфляции без тонкой настройки параметров); 3) демонстрируют наличие нескольких последовательных инфляционных фаз. Первое условие объясняется желанием качественного согласования механизма инфляции с физикой элементарных частиц, второе является необходимым ингредиентом теории инфляции, третье служит для согласования теории с данными современных наблюдений, свидетельствующими об ускоренном расширении Вселенной.
\end{abstract}

Ключевые слова: суперпотенциал, инфляция, уравнения Фридмана.

\section{1. ВВЕДЕНИЕ}

Одной из важнейших задач математической космологии является исследование различных сценариев генерации космологической инфляции и выхода из нее. Соответствующие модели содержат скалярное поле $\phi(t)$ (или набор полей) в однородном и изотропном пространстве-времени и в настоящее время используются не только для исследования процессов в ранней Вселенной, но и для описания современного ускоренного расширения Вселенной, обнаруженного по наблюдению сверхновых типа Іа в 1998 г. двумя группами астрономов [1].

Известно, что самосогласованная система уравнений Эйнштейна и минимально взаимодействующего скалярного поля с лагранжианом

$$
L=\frac{1}{2} \dot{\phi}^{2}-V(\phi)
$$

* Российский государственный университет им. И. Канта, Калининград, Россия. E-mail: yurov@freemail.ru

${ }^{\dagger}$ Department of Mathematics, University of Missouri, Columbia, USA

‡Ульяновский государственный педагогический университет, Ульяновск, Россия. E-mail: sv_chervon@rambler.ru

${ }^{\S}$ Centre for Theoretical Physics, Jamia Millia Islamia (A Central University), New Delhi, India 
(точка означает производную по времени) может быть представлена различными способами, так как мы имеем три уравнения при двух неизвестных функциях. Мы рассматриваем для простоты пространственно-плоскую модель однородной и изотропной Вселенной в метрике Фридмана-Робертсона-Уокера ${ }^{1)}$

$$
d s^{2}=d t^{2}-a^{2}(t) d l^{2}
$$

Система уравнений имеет вид

$$
\begin{gathered}
H^{2}=\frac{1}{2} \dot{\phi}^{2}+V(\phi), \\
\dot{H}=-\frac{3}{2} \dot{\phi}^{2}, \\
\ddot{\phi}+3 H \dot{\phi}+V^{\prime}(\phi)=0,
\end{gathered}
$$

где $\phi=\phi(t)$ - скалярное поле, $H=\dot{a} / a$ - параметр Хаббла, $a=a(t)$ - масштабный фактор. Любое из приведенных трех уравнений может быть получено как следствие (или дифференциальное следствие) оставшихся двух.

Существует несколько подходов интегрирования данной системы уравнений. В первую очередь стоит отметить прямой метод с заданным потенциалом. В качестве исходных двух уравнений выбираются уравнение Фридмана (1) и скалярного поля $\phi=\phi(t)(3)$ (в дальнейшем мы будем ссылаться на эту пару уравнений как на систему (1), (3)). Типичная постановка задачи выглядит следующим образом: проинтегрировать уравнения (1), (3) для заданного потенциала $V(\phi)$. Вид последнего обычно определяется предположениями, не связанными с космологией, а именно физикой элементарных частиц. В этом случае типичными являются модели свободного массивного поля $\left(V=m^{2} \phi^{2} / 2\right)$ или простейшие модели с самодействием $\left(V=\lambda \phi^{4} / 4\right)$ или, наконец, модели с потенциалом $V=m^{2} \phi^{2} / 2+\lambda \phi^{4} / 4$. К сожалению, во всех этих естественных с точки зрения физики высоких энергий моделях уравнения (1), (3) оказываются, как правило, неинтегрируемыми, и для проведения соответствующего анализа необходимо комбинировать численное изучение с разумными качественными оценками. По этой причине развитие получило направление математической космологии, связанное с изучением интегрируемых задач как упрощенных моделей инфляции. Однако большая часть таких моделей, как правило, демонстрирует неограниченную в будущем инфляцию, которая прекращается лишь при специальной подгонке параметров.

Вместе с тем успех инфляционных моделей [2]-[10], основанных на реалистичных потенциалах самодействия, создает впечатление, что основные эффекты - инфляция и выход из нее - слабо связаны с формой потенциала в точных уравнениях (1), (3). Во многом это убеждение основано на использовании приближения медленного скатывания, которое действительно демонстрирует наличие инфляции при самых общих предположениях. Часто полагают, что инфляция автоматически прекращается, как только приближение медленного скатывания перестает выполняться. Тем не менее многочисленные примеры существования инфляционного режима вне рамок приближения медленного скатывания можно найти в работах [11]-[19]2).

1) Здесь и далее мы пользуемся системой единиц, в которой $8 \pi G / 3=c=1$.

2) В работе [20] аналогично исследовались интегрируемые модели на бране и в объемлющем пространстве, снабженном структурой орбифолда. 
В работе [21] предложен новый подход к изучению уравнений (1), (3), основанный на сведении этой системы к уравнению Абеля первого рода. В качестве заданных потенциалов рассматривались степенные модели $\left(\phi^{2}, \phi^{4}\right)$. В частности, подробное исследование модели с квадратичным потенциалом в рамках указанного подхода подтвердило наличие естественного выхода из инфляции, который, однако, происходит несколько позднее нарушения условия медленного скатывания [21].

Аналогично ведут себя модели с потенциалом самодействия $\phi^{4}$. Более тщательный анализ показал, что в классе реалистичных начальных условий порядка $95 \%$ общего числа $e$-расширений (число расширений в $e$ раз) приходится, тем не менее, именно на область, в которой действует приближение медленного скатывания, поэтому обычные качественные оценки, использующие это приближение, корректны в классе указанных потенциалов (статья авторов на эту тему готовится к публикации). Общий итог этих исследований можно сформулировать так: строго говоря, нарушение приближения медленного скатывания является необходимым, но не достаточным условием прекращения инфляции, но для степенных потенциалов его можно использовать с хорошей точностью в качестве достаточного.

Основная задача настоящей работы - предложить эффективный метод построения интегрируемых потенциалов самодействия, которые удовлетворяют следующим условиям: 1) спонтанно нарушают симметрию; 2) описывают фазу инфляции с естественным выходом; 3) демонстрируют наличие нескольких последовательных инфляционных фаз. Первое условие мы рассматриваем как желательное, чтобы была возможность качественного согласования механизма инфляции с физикой элементарных частиц. Второе условие является необходимым ингредиентом теории инфляции. Третье условие служит для согласования теории с данными современных наблюдений.

Метод построения интегрируемых космологических моделей, удовлетворяющих условиям 1-3, уже частично использовался авторами этой статьи в работе [21], поэтому данную работу можно рассматривать как естественное продолжение исследования [21].

\section{2. МЕТОД СУПЕРПОТЕНЦИАЛА}

Идея рассматривать параметр Хаббла как функцию скалярных полей уже давно (свыше 20 лет) используется в космологии. В частности, этот подход весьма удобен при анализе уравнений Фридмана в формулировке Гамильтона-Якоби. Также как в космологии, так и в моделях с дополнительным пространственным измерением широко применяется метод суперпотенциала [22]-[34]. Суперпотенциал можно определять по-разному, например положить его равным параметру Хаббла. Мы, однако, зададим суперпотенциал $W$ как величину, равную квадрату параметра Хаббла (см. также [11]), т. е. в плоских космологических моделях ${ }^{3)}$

$$
W=\frac{\dot{\phi}^{2}}{2}+V
$$

3) Величину $W$ можно было бы назвать “гамильтонианом", однако мы сохраним термин суперпотенциал, поскольку его введение позволяет понизить порядок уравнения - типичное свойство моделей нерелятивистской суперсимметричной квантовой механики. 
и будем считать суперпотенциал $W$ зависящим только от величины скалярного поля $\phi: W=W(\phi)$. В этом случае система $(1),(3)$ примет простой вид:

$$
W^{\prime}(\phi)=-3 H \dot{\phi}, \quad H=\kappa \sqrt{W(\phi)},
$$

где $\kappa= \pm 1$.

Задавая суперпотенциал $W(\phi)$ (скажем, в виде полиномиальных выражений), мы одновременно определяем как потенциал самодействия (причем единственным образом)

так и динамику поля

$$
V=W-\frac{1}{18} \frac{W^{\prime 2}}{W}
$$

$$
\dot{\phi}=-\frac{1}{3 \kappa} \frac{W^{\prime}}{\sqrt{W}},
$$

которая уже может определяться неоднозначно, в зависимости от величин параметров, входящих в правую часть (7). Тем не менее величина $\phi(t)$ определяется простой квадратурой с последующим обращением функции $t(\phi)$. Подставляя найденную величину $\phi(t)$ во второе уравнение системы (5), находим параметр Хаббла и масштабный фактор однократным интегрированием. В дальнейшем ограничимся случаем $\kappa=+1$.

Основной результат данной работы основан на следующем утверждении: выбор суперпотенциала (4) в виде четной, положительно определенной и растущей полиномиальной функции позволяет определить потенциалы (6), удовлетворяющие условиям 1-3. Общая идея может быть проиллюстрирована на следующем примере.

Рассмотрим суперпотенциал в виде

$$
W=\frac{\lambda \phi^{n}}{n},
$$

где $\lambda>0, n>0$. Подставляя (8) в (6), находим

$$
V=\lambda \phi^{n-2}\left(\frac{\phi^{2}}{n}-\frac{n}{18}\right) .
$$

При четном $n>2$ потенциал (9) спонтанно нарушает симметрию (условие 1 ). При этом два симметричных минимума расположены в точках

$$
\phi_{ \pm}= \pm \frac{\sqrt{2 n(n-2)}}{6},
$$

при этом

$$
V\left(\phi_{ \pm}\right)=-\frac{2 \lambda}{n(n-2)}\left(\frac{2 n(n-2)}{36}\right)^{n / 2} .
$$

Интегрируя (7) и действуя по указанной выше схеме, находим

$$
\phi(t) \sim\left[\sqrt{\lambda n}(n-4)\left(t-t_{*}\right)\right]^{2 /(4-n)},
$$

где $t_{*}$ - постоянная интегрирования, $n \neq 4$ (случай $n=4$ будет подробно рассмотрен ниже). Анализируя полученное выражение, можно прийти к следующему заключению: при $0<n<4$ режим медленного скатывания выполняется при $\left|t_{*}-t\right| \rightarrow \infty$ 
и нарушается при $t \rightarrow t_{*}$. Если $n>4$, то режим медленного скатывания, напротив, выполняется при малых величинах разности $t-t_{*}$ и нарушается при $t \rightarrow \infty$. В обоих случаях можно ожидать наличия режима инфляции с естественным выходом, т. е. выполнения условия 2. Далее мы подробно рассмотрим случай четных потенциалов с показателем, не превышающим ${ }^{4)} 4$.

\section{3. ОТ $W(\phi)$ K $V(\phi)$ CO СПОНТАННО НАРУШЕННОЙ СИММЕТРИЕЙ (УСЛОВИЕ 1)}

Наиболее общий вид суперпотенциала, отвечающего нашим требованиям, имеет вид

$$
W=\frac{\lambda \phi^{4}}{4}+\frac{m^{2} \phi^{2}}{2}+\Lambda,
$$

причем константы $\lambda>0, \Lambda>0$. Используя (6), можно вычислить потенциал самодействия $V(\phi)$ и исследовать его. Величина $V(\phi)$ обладает следующими свойствами: это четная функция, имеющая асимптотическое поведение $V(\phi) \rightarrow \lambda \phi^{4} / 4$ при $|\phi| \rightarrow \infty$ и точку экстремума при $\phi=0$. При выполнении условия

$$
\Lambda<\frac{m^{2}}{9}
$$

точка $\phi=0$ оказывается точкой локального максимума и появляются еще два симметрично расположенных минимума. Другими словами, справедливость неравенства (11) гарантирует наличие спонтанно нарушенной симметрии у потенциала $V(\phi)$, т. е. выполнение условия $1^{5)}$.

Как будет показано ниже, физический смысл величины $\Lambda$ - космологическая постоянная, которая проявляет себя лишь на поздней стадии расширения вселенной. По этой причине при анализе модели, основанной на суперпотенциале (10), на предмет наличия инфляции с естественным выходом (условие 2) константу $\Lambda$ можно опустить. Мы восстановим ее в разделе 4 , а пока будем всюду полагать $\Lambda=0$.

Итак, подставляя (10) с $\Lambda=0$ в (6), находим

$$
V=\frac{\lambda \phi^{4}}{4}+\frac{m^{2} \phi^{2}}{2}-\frac{2\left(\lambda \phi^{2}+m^{2}\right)^{2}}{9\left(\lambda \phi^{2}+2 m^{2}\right)} .
$$

Исследуем этот потенциал. Во-первых, при больших по модулю значениях $\phi$ этот потенциал практически сводится к модели с потенциалом самодействия $\phi^{4}$ с константой связи $\lambda$. Во-вторых, потенциал (12) имеет критические точки, определяемые соотношениями

$$
\phi=0, \quad \phi_{ \pm}^{2}=\frac{1}{\lambda}\left[-2 m^{2}+\frac{2 \lambda}{9} \pm \frac{2}{9} \sqrt{9 \lambda m^{2}+\lambda^{2}}\right] .
$$

\footnotetext{
4) $\mathrm{C}$ точки зрения квантовой теории поля более высокие степени будут приводить к неперенормируемым, а значит, малоинтересным моделям с $D=4$.

${ }^{5)}$ Хотя неравенство (11) выполняется при $\Lambda=0, m \neq 0$, первый член разложения потенциала самодействия $V(\phi)$ в ряд Тейлора по степеням $\phi$ оказывается расходящимся при $\Lambda \rightarrow 0$, поэтому случай $\Lambda=0$ требует отдельного рассмотрения.
} 


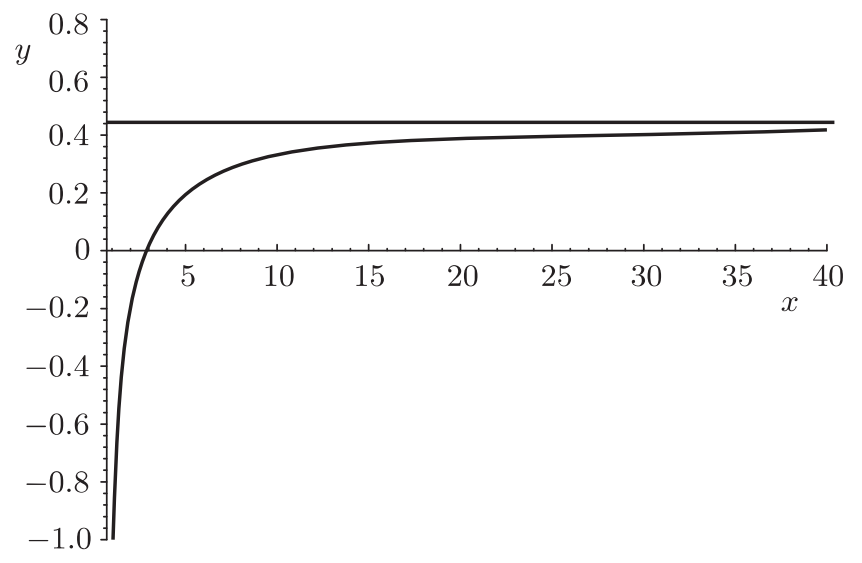

Рис. 1. Зависимость $\phi_{+}^{2}(x)$.

Величина $\phi_{+}^{2}>0$, если $\lambda>3 m^{2}$. Удобно ввести безразмерную переменную $x=$ $\lambda / m^{2}$. При этом

$$
\phi_{+}^{2}=\frac{2(-9+x+\sqrt{x(9+x)})}{9 x} .
$$

График $\phi_{+}^{2}(x)$ изображен на рис. 1 , причем точка пересечения с осью абсцисс имеет место при критическом значении $x=3$. Что же касается величины

$$
\phi_{-}^{2}=\frac{2(-9+x-\sqrt{x(9+x)})}{9 x},
$$

то она оказывается отрицательной при $m^{2}>0, \lambda>0$ (при $x \rightarrow \infty, \phi_{-}^{2} \rightarrow 0$, причем $\left.d \phi_{-}^{2} / d x>0\right)$ и по этой причине не представляет интереса.

Определим массу скалярного поля обычным образом:

$$
M^{2}=\left(\frac{d^{2} V}{d \phi^{2}}\right)_{\phi=0}=m^{2}\left(1-\frac{x}{3}\right)=m^{2}-\frac{\lambda}{3} .
$$

Таким образом, при $0<x<3$ величина $M^{2}>0, \phi_{+}^{2}<0$ и симметрия точна. Если же $x>3$, то $M^{2}<0$ и симметрия спонтанно нарушена. Случай $x=0$ отвечает нулевой константе связи, а случай $x=3$ описывает безмассовое поле с потенциалом

$$
\frac{V}{m^{2}}=\frac{3 \phi^{4}}{4}-\frac{\phi^{2}}{6}-\frac{2}{9\left(3 \phi^{2}+2\right)}
$$

и ненарушенной симметрией (см. рис. 2).

Более интересны потенциалы, нарушающие симметрию (см. рис. 3, где изображен потенциал для $x=10$ ). Два симметричных минимума расположены в точках

$$
\phi_{ \pm}= \pm \sqrt{\frac{2(-9+x+\sqrt{x(9+x)})}{9 x}}
$$




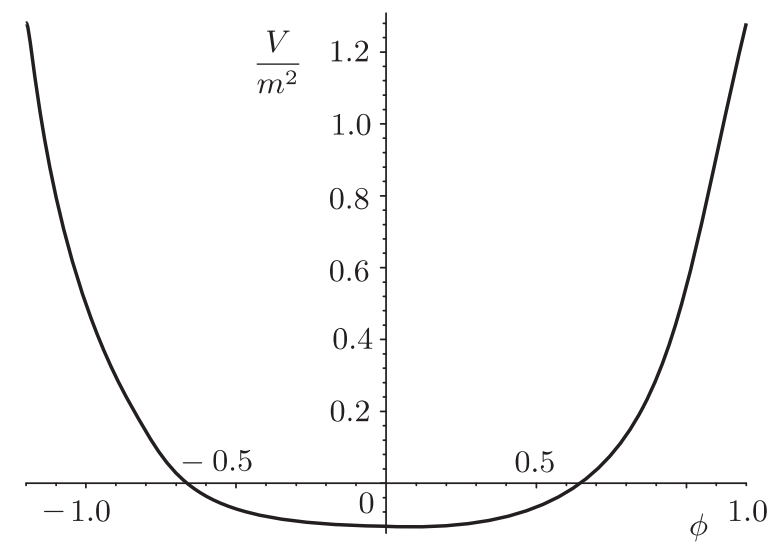

Рис. 2. Потенциал (15), отнесенный к квадрату “массы” $\mathrm{m}^{2}$.

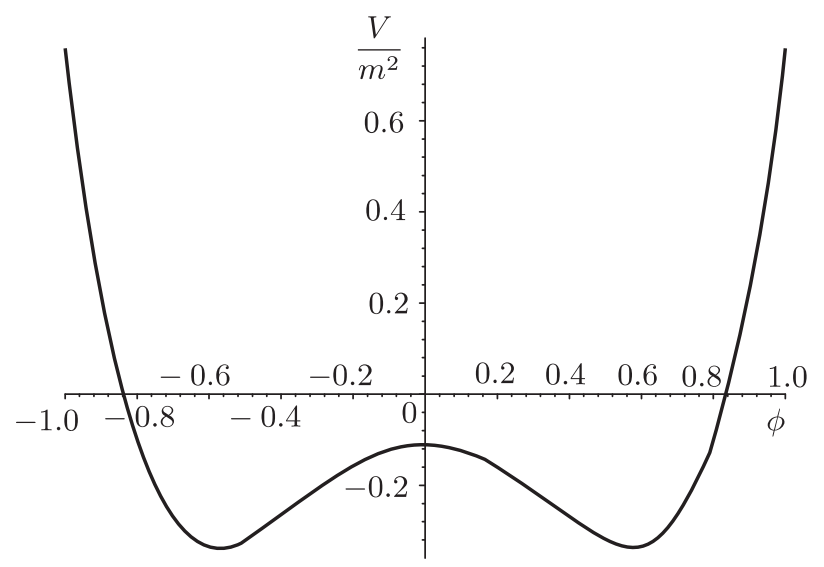

Рис. 3. Потенциал (12), отнесенный к квадрату "массы" $\mathrm{m}^{2}$, при $\lambda=10 \mathrm{~m}^{2}$.

причем значение потенциала в этих точках

$$
V_{\min }=-\frac{m^{2}\left(4 x^{2}+(4 x-27) \sqrt{x(9+x)}-9 x+162\right)}{81(x+\sqrt{x(9+x)})} .
$$

При $|x| \gg 1$ имеем $V_{\min } \sim-4 \lambda / 81$. Поскольку $V(0)=-m^{2} / 9$, то при достаточно большой величине $\lambda$ и достаточно малой величине $m^{2}$ потенциал (12) будет с хорошей точностью аппроксимироваться обычным хиггсовским потенциалом, обращающимся в нуль при $\phi=0$.

Отметим, что такой потенциал можно получить в другом предельном случае $m=0$. При этом потенциал (12) принимает привычный вид:

$$
V=\frac{\lambda \phi^{4}}{4}-\frac{2 \lambda \phi^{2}}{9}
$$

Здесь имеет место тонкая настройка по массе: $M^{2}=-4 \lambda / 9$, а точки минимума лежат при $\phi_{ \pm}= \pm 2 / 3$. 


\section{4. ИНФЛЯЦИЯ С ВЫХОДОМ (УСЛОВИЕ 2)}

4.1. Общая модель (12). Интегрируя уравнения (5) с потенциалом (12), находим решение в параметрическом виде:

$$
\begin{gathered}
t=C-\frac{3}{2 \sqrt{\lambda}}\left[\ln \left|\operatorname{ctg} \frac{\xi}{2}\right|+\frac{1}{2} \operatorname{arctg}(\cos \xi)\right], \\
a=a_{*}\left(\frac{\sin ^{2} \xi}{2-\sin ^{2} \xi}\right)^{3 m^{2} / 8 \lambda} \exp \left(-\frac{3 m^{2}}{4 \lambda \sin ^{2} \xi}\right), \\
\phi=-\frac{m \sqrt{2}}{\sqrt{\lambda}} \operatorname{ctg} \xi, \quad H=\frac{m^{2}}{\sqrt{\lambda}} \frac{\cos \xi}{\sin ^{2} \xi} .
\end{gathered}
$$

Здесь параметр $\xi$ принадлежит интервалу $(0, \pi)$, что соответствует $t \in(-\infty,+\infty)$, а $C$ и $a_{*}-$ постоянные интегрирования. Вселенная, описываемая системой (17), расширяется на полуоси $t \in(-\infty, 0)$ и сжимается при $t \in(0,+\infty)$. Другими словами, решение описывает вселенную, начинающую и заканчивающую свое существование в точке сингулярности при $t=\mp \infty$, однако в рамках классического описания ее существование можно считать конечным по времени, ограниченным моментами, при которых масштабный фактор становится равен планковскому масштабу $a_{\mathrm{Pl}}=10^{-33} \mathrm{~cm}$.

Параметр ускорения, выраженный через введенную в предыдущем разделе безразмерную величину $x$, имеет вид

$$
\frac{\ddot{a}}{a}=-\frac{m^{2}}{3 x \sin ^{4} \xi}\left(x \sin ^{6} \xi-4 x \sin ^{4} \xi+(4 x+3) \sin ^{2} \xi-3\right) .
$$

На рис. 4 представлен график величины (18) при $x=3$ (это отвечает безмассовому полю с ненарушенной симметрией, см. (14)). Как следует из приведенного графика, на ранней стадии вселенная переживает инфляцию с естественным выходом (для

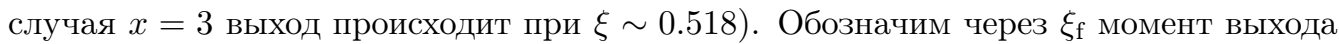
из инфляции и будем считать, что $\xi_{\mathrm{f}} \ll 1$. Оставляя главные вклады в $(18)$, находим удобные формулы:

$$
\xi_{\mathrm{f}} \sim \sqrt{\frac{3}{4 x}}, \quad t_{\mathrm{f}} \sim C-\frac{3}{4 m \sqrt{x}}\left(\ln \frac{16 x}{3}-\frac{\pi}{2}\right) .
$$

Что касается второй фазы положительного ускорения, то она реализуется уже на стадии сжатия вселенной и не представляет для нас интереса.

Решение (17) не допускает гладкого перехода при $\lambda=0$ и $m^{2}=0$, поэтому целесообразно рассмотреть эти случаи отдельно.

4.2. Случай $W=\lambda \phi^{4} / 4$. Интегрируя уравнения (5), находим

$$
\begin{aligned}
& a(t)=a_{0} \exp \left[\frac{3 \phi_{0}^{2}}{8}\left(1-e^{-4 \sqrt{\lambda}\left(t-t_{0}\right) / 3}\right)\right], \\
& \phi(t)=\phi_{0} \exp \left[-\frac{2 \sqrt{\lambda}}{3}\left(t-t_{0}\right)\right], \\
& H(t)=\frac{\sqrt{\lambda} \phi_{0}}{2} \exp \left[-\frac{4 \sqrt{\lambda}}{3}\left(t-t_{0}\right)\right],
\end{aligned}
$$




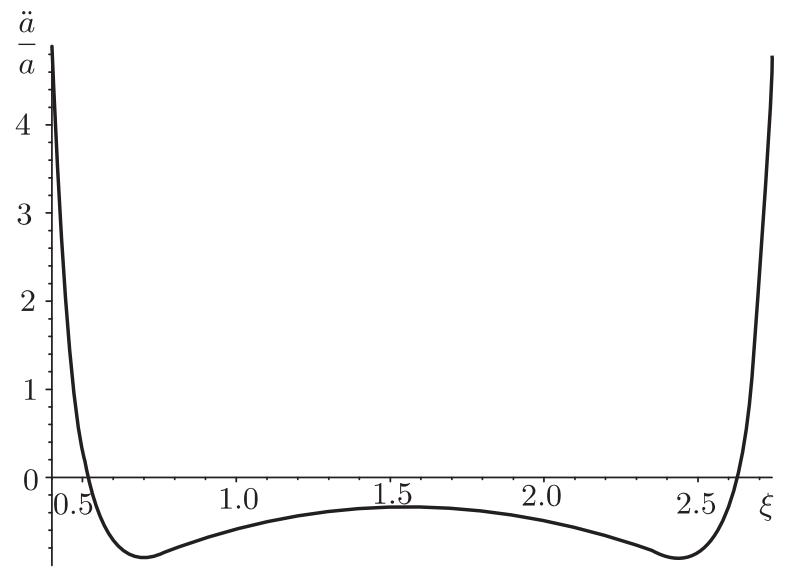

Рис. 4. График параметра ускорения (18) при $x=3$ и $m^{2}=1$.

где $\phi_{0}, a_{0}$ и $t_{0}$ - постоянные интегрирования. Модель (19) описывает инфляцию на ранней стадии, которая заканчивается в момент времени $t=t_{\mathrm{f}}$, когда масштабный фактор достигает значения $a=a_{\mathrm{f}}$ :

$$
t_{\mathrm{f}}=t_{0}+\frac{3}{4 \sqrt{\lambda}} \ln \left(\frac{3 \phi_{0}^{2}}{8}\right), \quad a_{\mathrm{f}}=a_{0} \exp \left(\frac{3 \phi_{0}^{2}}{8}-1\right) .
$$

Если условно принять момент $t=t_{0}$ за начало инфляции, то число $e$-расширений

$$
N_{\mathrm{f}}=\frac{3 \phi_{0}^{2}}{8}-1
$$

Например, полагая $N_{\mathrm{f}}=60$, получаем $\phi_{0}=12.75$, а значение $N_{\mathrm{f}}=100$ соответствует $\phi_{0}=16.41$.

4.3. Случай $W=m^{2} \phi^{2} / 2$. Решение уравнений (5) имеет вид

$$
\begin{aligned}
a(t) & =a_{0} \exp \left[-\frac{m}{6}\left(t-t_{0}\right)\left(m\left(t-t_{0}\right)-3 \sqrt{2} \phi_{0}\right)\right], \\
\phi(t) & =\phi_{0}-\frac{\sqrt{2} m}{3}\left(t-t_{0}\right), \\
H(t) & =\frac{m \phi_{0}}{\sqrt{2}}-\frac{m^{2}}{3}\left(t-t_{0}\right) .
\end{aligned}
$$

Как и в двух предыдущих случаях, это решение описывает вселенную, начинающую и заканчивающую свое существование в точке сингулярности при $t=\mp \infty$. Параметр ускорения

$$
\frac{\ddot{a}}{a}=\frac{m^{2}}{18}\left(2 m^{2} t^{2}-2 m\left(3 \sqrt{2} \phi_{0}+2 m t_{0}\right) t+2 m^{2} t_{0}^{2}+6 m t_{0} \sqrt{2} \phi_{0}+9 \phi_{0}^{2}-6\right) .
$$

Опять условно выберем $t_{0}$ в качестве момента начала инфляции, при котором масштабный фактор $a_{0}>a_{\mathrm{Pl}}$ (для этого необходимо, чтобы $\phi_{0}>\sqrt{2 / 3}$ ). Инфляция на 
стадии расширения прекращается в момент

$$
t_{\mathrm{f}}=t_{0}-\frac{3}{m}+\frac{3 \sqrt{2} \phi_{0}}{2 m}
$$

Число e-расширений

$$
N_{\mathrm{f}}=\frac{3 \phi_{0}^{2}}{4}-1
$$

а продолжительность инфляции

$$
t_{\mathrm{f}}-t_{0}=\frac{3 \phi_{0}-\sqrt{6}}{m \sqrt{2}}=\frac{\sqrt{3}}{m}\left(\sqrt{1+2 N_{\mathrm{f}}}-1\right) .
$$

Отметим еще, что расширение сменяется сжатием в момент $t_{\mathrm{stop}}=t_{0}+3 \sqrt{2} \phi_{0} / 2 \mathrm{~m}$, так что $t_{\text {stop }}-t_{\mathrm{f}}=\sqrt{3} / \mathrm{m}$.

4.4. Модели с космологической постоянной (условие 3). Хотя все предыдущие примеры демонстрировали наличие инфляции с естественным выходом, они не приводили ко второй фазе ускоренного расширения, которую наша Вселенная переживает в настоящее время. Для того чтобы получить соответствующую модель, выберем суперпотенциал (10) с $\lambda=0$ :

$$
W=\frac{m^{2} \phi^{2}}{2}+\Lambda
$$

Решение удобно выразить через полевую переменную $\phi$ :

$$
\begin{gathered}
t=t_{*}+\frac{3}{2 m^{2}}\left[2 \sqrt{\Lambda} \ln \left(\frac{\sqrt{2 \Lambda}}{\phi}\left(\sqrt{2 \Lambda}+\sqrt{m^{2} \phi^{2}+2 \Lambda}\right)\right)-\sqrt{2 m^{2} \phi^{2}+4 \Lambda}\right], \\
a=a_{*} \phi^{-3 \Lambda / m^{2}} e^{-3 \phi^{2} / 4} \\
H=\sqrt{\frac{m^{2} \phi^{2}+2 \Lambda}{2}} .
\end{gathered}
$$

При этом поле $\phi(t) \rightarrow 0$ при $t \rightarrow+\infty$ и $\phi(t) \rightarrow+\infty$ при $t \rightarrow-\infty$. Используя (22), находим параметр ускорения:

$$
\frac{\ddot{a}}{a}=\frac{3 m^{4}\left(\phi^{2}-\phi_{+}^{2}\right)\left(\phi^{2}-\phi_{-}^{2}\right)}{6\left(m^{2} \phi^{2}+2 \Lambda\right)},
$$

где

$$
\phi_{ \pm}^{2}=-\frac{2 \Lambda}{m^{2}}+\frac{1}{3} \pm \frac{\sqrt{m^{2}-12 \Lambda}}{3 m} .
$$

Из (23) следует, что при $\phi \rightarrow \infty$ (ранняя вселенная) $\ddot{a} / a \rightarrow m^{2} \phi^{2} / 2$, а на поздней стадии при $\phi \rightarrow 0$ имеем $\ddot{a} / a \rightarrow \Lambda$, откуда вытекает, что величина $\Lambda$ является "настоящей" космологической постоянной. Общая динамика вселенной, описанной формулами (22), выглядит следующим образом: при $\phi>\phi_{+}$вселенная переживает стадию инфляционного расширения, при $\phi_{-}<\phi<\phi_{+}$она расширяется без ускорения, а при $\phi<\phi_{-}$находится в фазе второй, более медленной инфляции, обусловленной наличием космологической постоянной. 
Рассмотрим более подробно первую фазу инфляции. Полагая $\Lambda \ll m^{2}$, получаем окрестность точки $\phi_{+}^{2}$ :

$$
\phi_{+} \sim \frac{\sqrt{6}}{3}, \quad t_{\mathrm{f}} \sim t_{*}-\frac{\sqrt{3}}{m}+\frac{3 \sqrt{\Lambda}}{m^{2}} \ln (m \sqrt{3 \Lambda}),
$$

а число $e$-расширений

$$
N_{\mathrm{f}}=\frac{3 \Lambda}{2 m^{2}} \ln \left(\frac{\phi_{\mathrm{i}}^{2}}{\phi_{+}^{2}}\right)+\frac{3}{4}\left(\phi_{+}^{2}-\phi_{\mathrm{i}}^{2}\right),
$$

где $\phi_{\mathrm{i}}$ - значение поля, которое мы, как и выше, условно отождествляем с началом инфляции. Длительность фазы инфляции будет мала по сравнению с промежутком времени неускоренного расширения, если

$$
\phi_{\mathrm{i}} \ll \frac{2 \sqrt{6}}{3}+\frac{\sqrt{2 \Lambda} \ln m^{2}}{m} .
$$

Теперь, дифференцируя давление по плотности, вычислим квадрат скорости звука, который оказывается равным

$$
c_{\mathrm{s}}^{2}=\frac{9 m^{2}\left(\phi^{2}+\left|\phi_{\mathrm{cs}}^{(+)}\right|^{2}\right)\left(\left(\phi_{\mathrm{cs}}^{(-)}\right)^{2}-\phi^{2}\right)}{9\left(m^{2} \phi^{2}+2 \Lambda\right)^{2}},
$$

где

$$
\left(\phi_{\mathrm{cs}}^{( \pm)}\right)^{2}=-\frac{2 \Lambda}{m^{2}} \mp \frac{2 \sqrt{2 \Lambda}}{3 m} .
$$

Используя эти соотношения, можно показать, что условие $\Lambda<2 m^{2} / 9$ гарантирует вещественность $\phi_{\mathrm{cs}}^{(-)}$и устойчивость расширения по отношению к малым возмущениям на поздней стадии расширения (в частности, во время второй фазы инфляции, которую переживает Вселенная в настоящее время). Соответственно, на ранней стадии, при $\phi>\phi_{\mathrm{cs}}^{(-)}$, имеет место рост неустойчивости.

В заключение приведем явный вид потенциала самодействия:

$$
V=\frac{m^{2} \phi^{2}}{2}+\Lambda-\frac{m^{4} \phi^{2}}{9 m^{2} \phi^{2}+18 \Lambda},
$$

и при $\phi \rightarrow 0$ имеем $V \rightarrow \Lambda$, как и должно быть. Квадрат массы определяется выражением

$$
M^{2}=m^{2}-\frac{m^{4}}{9 \Lambda}
$$

При $\Lambda<m^{2} / 9$ потенциал (24) спонтанно нарушает симметрию, обладая двумя минимумами при

$$
\phi_{*}^{2}=\frac{2(m \sqrt{\Lambda}-3 \Lambda)}{3 m^{2}} .
$$

Выбирая правый минимум и переходя к новой полевой переменной $\Phi$ путем преобразования Боголюбова $\phi=\Phi+\phi_{*}$, получаем окончательно

$$
V=\frac{m Z}{108}+\frac{12 m \Lambda}{Z}-\frac{m^{2}}{9}
$$


где

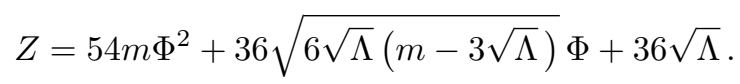

При этом вещественная масса поля $\Phi$

$$
M_{\Phi}^{2}=4 m(m-3 \sqrt{\Lambda}) .
$$

\section{5. ЗАКЛЮЧЕНИЕ}

Мы показали, что фиксация суперпотенциала как полиномиальной функции поля $W=W(\phi)$ позволяет неожиданно просто строить интегрируемые модели со спонтанно нарушенной симметрией, описывающие инфляцию и естественный выход из нее, без необходимости "тонкой настройки" параметров. Указанный подход может оказаться полезным и в более экзотических областях, связанных, например, с изучением фантомной составляющей. В частности, можно показать, что требование мнимости полевой переменной $\phi$, но вещественности суперпотенциала достаточно для построения фантомных лагранжианов, демонстрирующих весьма богатый репертуар поведения, в том числе эффект пересечения фантомной зоны. Этот вопрос будет рассмотрен в отдельной публикации.

Благодарности. Работа осуществлена при частичной финансовой поддержке программы Российско-Индийского сотрудничества РФФИ (грант № 08-02-91307ИНД_а). А.В. Юров благодарит РФФИ (грант № 09-05-00446а) и Европейский научный фонд (грант FIS2006-02842) за финансовую поддержку. Авторы выражают искреннюю благодарность рецензенту, чьи критические замечания во многом способствовали улучшению качества статьи.

\section{Список литературы}

[1] S. J. Perlmutter, G. Aldering, G. Goldhaber et al., Astrophys. J., 517:2 (1999), 565-586, arXiv: astro-ph/9812133; N. A. Bachall, J. P. Ostriker, S. Perlmutter, P. J. Steinhardt, Science, 284:5419 (1999), 1481-1488, arXiv: astro-ph/9906463; A. G. Riess, A. V. Filippenko, P. Challis et al. (Team Collab.), Astron. J., 116:3 (1998), 1009-1038, arXiv: astro-ph/9805201; V. Sahni, A. A. Starobinsky, Internat. J. Modern Phys. D, 9:4 (2000), 373-443, arXiv: astro-ph/9904398.

[2] А. А. Старобинский, Писъма в ЖЭЭФ, 30:11 (1979), 719-723; А. A. Starobinsky, Phys. Lett. B, 91:1 (1980), 99-102.

[3] A. Guth, Phys. Rev. D, 23:2 (1981), 347-356.

[4] A. D. Linde, Phys. Lett. B, 108:6 (1982), 389-393.

[5] A. Albrecht, P. J. Steinhardt, Phys. Rev. Lett., 48:17 (1982), 1220-1223.

[6] В. Ф. Муханов, Г. В. Чибисов, Писъма в ЖЭТФ, 33:10 (1981), 549-553.

[7] S. W. Hawking, Phys. Lett. B, 115:4 (1982), 295-297.

[8] A. A. Starobinsky, Phys. Lett. B, 117:3-4 (1982), 175-178.

[9] V.F. Mukhanov, H. A. Feldman, R. H. Brandenberger, Phys. Rep., 215:5-6 (1992), 203-333.

[10] A. D. Linde, Phys. Lett. B, 129:3-4 (1983), 177-181.

[11] В. М. Журавлев, С. В. Червон, В. К. Щиголев, ЖЭТФ, 114:2 (1998), 406-417.

[12] J. D. Barrow, Phys. Rev. D, 49:6 (1994), 3055-3058.

[13] R. Maartens, D. R. Taylor, N. Roussos, Phys. Rev. D, 52:6 (1995), 3358-3364.

[14] G.F. R. Ellis, M. S. Madsen, Class. Quantum Grav., 8:4 (1991), 667-676. 
[15] J. E. Lidsey, Class. Quantum Grav., 8:5 (1991), 923-933.

[16] J. D. Barrow, P. Saich, Class. Quantum Grav., 10:2 (1993), 279-283.

[17] P. Parson, J. D. Barrow, Class. Quantum Grav., 12:7 (1995), 1715-1721.

[18] A.V. Yurov, Phantom scalar fields result in inflation rather than Big Rip, arXiv: astro-ph/0305019.

[19] С. Д. Верещагин, А. В. Юров, ТМФ, 139:3 (2004), 405-422.

[20] A. V. Yurov, V. A. Yurov, Phys. Rev. D, 72:2 (2005), 026003, 12 pp., arXiv: hep-th/0412036.

[21] A. V. Yurov, V. A. Yurov, J. Math. Phys., 51:8 (2010), 082503, 17 pp.

[22] A. G. Muslimov, Class. Quantum Grav., 7:2 (1990), 231-237.

[23] D. S. Salopek, J. R. Bond, Phys. Rev. D, 42:12 (1990), 3936-3962.

[24] D. Bazeia, M. J. dos Santos, R. F. Ribeiro, Phys. Lett. A, 208:1-2 (1995), 84-88, arXiv: hep-th/0311265.

[25] A. Brandhuber, K. Sfetsos, JHEP, 10 (1999), 13, 20 pp., arXiv: hep-th/9908116.

[26] O. DeWolfe, D. Z. Freedman, S. S. Gubser, A. Karch, Phys. Rev. D, 62:4 (2000), 046008, 16 pp., arXiv: hep-th/9909134.

[27] D. Bazeia, C. B. Gomes, L. Losano, R. Menezes, Phys. Lett. B, 633:4-5 (2006), 415-419, arXiv: astro-ph/0512197.

[28] K. Skenderis, P.K. Townsend, Phys. Rev. D, 74:12 (2006), 125008, 10 pp., arXiv: hep-th/0609056.

[29] D. Bazeia, L. Losano, J. J. Rodrigues, First-order formalism for scalar field in cosmology, arXiv: hep-th/0610028.

[30] И. Я. Арефьева, С. Ю. Вернов, А. С. Кошелев, ТМФ, 148:1 (2006), 23-41.

[31] I. Ya. Aref'eva, A. S. Koshelev, S. Yu. Vernov, Phys. Rev. D, 72:6 (2005), 064017, 11 pp., arXiv: astro-ph/0507067.

[32] A. S. Mikhailov, Yu. S. Mikhailov, M. N. Smolyakov, I. P. Volobuev, Class. Quantum Grav., 24:1 (2007), 231-242, arXiv: hep-th/0602143.

[33] С. Ю. Вернов, ТМФ, 155:1 (2008), 47-61, arXiv: astro-ph/0612487.

[34] P. K. Townsend, Class. Quantum Grav., 25:4 (2008), 045017, 16 pp., arXiv: 0710.5178.

Поступила в редакцию 15.05.2010, после доработки 18.08.2010 